\title{
A Descriptive Study of Histopathological Patterns of Lymph Node Biopsies In A Tertiary Care Hospital
}

\author{
Rajshri P. Damle*, Kishor H. Suryawanshi, N.V.Dravid, D.V.Newadkar and Prashant N. Deore \\ Department Of Pathology, A.C.P.M. Medical College, Dhule, Maharashtra. India.
}

\section{ABSTRACT}

Background: Lymph node is affected by various types of lesions, both non-neoplastic (inflammatory) or neoplastic. Due to non-specific symptoms, the clinical diagnosis are usually delayed thus lymph node biopsy is mandatory to arrive at specific diagnosis for appropriate management.

Objective: 1) To study the spectrum of lymph node pathology with clinical correlation.

2) To study the different histological patterns of lymph node lesions and classify them into major categories.

Methods: The present study was a prospective observational type of study and included 331 patients who presented with lymph node enlargement in a tertiary care hospital over a period of 3 years from Jan 2013 to Dec 2015. Detailed clinical history was noted. The specimens were routinely processed and stained by Haematoxylin and eosin. Special stains along with immunohistochemistry were done whenever required.

Result: A total of 331 lymph node biopsies were studied. Age distribution varied from 4 to 81 years with male to female ratio of 1:1.4. Non -neoplastic lesions comprised of maximum cases (80.06\%) while neoplastic lesion were present in (19.93\%) cases. Reactive lymphadenitis was the predominant non-neoplastic finding followed by granulomatous lymphadenitis. Neoplastic lesions were included $3.61 \%$ cases of lymphoma and $16.31 \%$ cases of metastatic lesions.

Conclusion: Lymphadenopathy is not uncommon in our region so lymph node biopsy is an important tool for early diagnostic and prognostic purpose. Reactive lymphadenitis was the most common cause of lymphadenopathy followed by granulomatous lymphadenitis.

Keywords: Lymphadenopathy, Biopsy, Histopathological Examination, Diagnostic

\section{Introduction}

Lymph nodes are the most widely distributed and easily accessible lymphoid tissue and are frequently examined for diagnostic purpose. The degree and pattern of the morphologic changes are dependent on the inciting stimulus and the intensity of the response. Trivial injuries and infections induce subtle changes while more significant infections inevitably produce nodal enlargement. ${ }^{[1]}$

Lymphadenopathy is a common clinical condition which may be neoplastic or non-neoplastic, affects any age group and can be diagnosed on clinical history, physical examination and fine needle aspiration cytology (FNAC). FNAC is commonly used to establish the etiological diagnosis of lymph node lesions but the limitation of this technique is unable to diagnose suspected or grey zone of various lesions and lymphohematogenous malignancy. So excision biopsy and histopathological examination of the lymph node remains the 'Gold standard' for diagnosis. ${ }^{[2,3]}$

Hence this study was undertaken to study the different histological patterns in detail of lymph node biopsies.

\section{Materials and Methods}

The present study was carried out in Department of pathology ACPM Medical College Dhule from Jan 2013 to Dec 2015 and included 331 cases of lymph node biopsies. Detailed clinical history like age, sex, site, presenting complaints and clinical diagnosis was noted. Relevant findings and investigations were recorded. The study was approved by institutional ethical committee.

\section{Eligibility criteria adopted in our study:}

1) Inclusion criteria: all type of lymph node specimens and all age groups.

2) Exclusion criteria: inadequate samples and poorly preserved tissue were excluded.

All cases of lymph node biopsies were received in 10\% formalin. The gross morphological features like size of node, shape, color, consistency, presence of necrosis and matting were noted and taken sections from each. The specimens were processed in automated tissue processor and 4-5 micron thick paraffin embedded sections were taken and stained by Haematoxylin and 
eosin. Special stain including Ziehl Neelsen, periodic acid Schiff (PAS) and reticulin were used where indicated. Immunohistochemistry was performed whenever required. Each slide was carefully examined by two pathologist and the histopathological patterns of lymph node biopsies were reported and categorized.

\section{Results}

In the present study, a total of 331 lymph node biopsies were received to the department of pathology and reviewed cases were categorized into four main groups; 175 cases (52.87\%) of reactive lymphadenitis, 82 cases $(24.77 \%)$ of granulomatous lymphadenitis, 66 cases $(19.93 \%)$ of neoplastic disease and 8 cases $(2.41 \%)$ of miscellaneous group . [Table- 1]

Out of these, 135 (40.78\%) were males and 196 (59.21\%) were females giving a male to female ratio was 1:1.4. Most of the cases (78\%) presented with clinical features of pain and tenderness. Age group of patients ranged from 4 years to 81 years. Maximum no. of patients were in the age group of $21-30$ years $(22.05 \%)$ followed by $31-40$ years $(18.42 \%)$ and least cases were seen in the age group over 70 years $(2.11 \%)$ [Table -2].

In the present study, non-neoplastic lesions were more common comprising 265 cases $(80.06 \%)$ and neoplastic lesions were 66 cases $(19.93 \%)$. The most common sites were observed from the cervical lymph node followed by axillary lymph node representing $(65.86 \%)$ and $(15.70 \%)$ respectively. [Fig.1]

Among non-neoplastic lesions, reactive lymphadenitis were the most common accounting for 175 cases $(52.87 \%)$ and maximum number of cases was observed in 21-30 yrs age group. Out of 175 cases, chronic non-specific lymphadenitis $(36.85 \%)$ was more common followed by follicular hyperplasia in (9.96\%)[Fig.2d], sinus histiocytosis in (3.62\%) [Fig.3a] and paracortical hyperplasia in (2.41\%). Majority of cases of chronic non-specific lymphadenitis were in the age group of 21-30 yrs followed by 31-40 yrs of age group.

In our study, granulomatous lymphadenitis was the second most common cause of lymphadenopathy. Tuberculosis was the most frequent cause accounting for 67 cases (20.24\%) of granulomatous lesions [Fig-2a]. The most common age group was 31-40 years of age with slight female predominance. AFB was positive in 46 cases (68.5\%) [Fig-2b]. Other 15 cases of granulomatous lesions included only 2 cases of cat scratch disease and 13 cases were not otherwise specified or categorized.

Among neoplastic lesions, primary neoplasm of lymph nodes were 12 cases $(3.61 \%)$. Out of which 4 cases of Hodgkin's lymphoma [Fig-3b] were diagnosed and in which 3 cases were observed in 41-60 years of age group and only single case was observed in 20 years of female patient. There were 8 cases of Non-Hodgkin's lymphoma and diagnosed after the age group of 30 years and peak between 41-50 years with slight male predominance. Out of 8 cases, 5 cases were diffuse small lymphocytic lymphoma [Fig-3d] and 3 cases were follicular lymphoma [Fig-3c]. All cases of primary neoplasm of lymph node were confirmed by immunohistochemistry.

Secondary neoplasm of lymph node were 54 cases $(16.31 \%)$ in which 15 were male and 39 were female patients and most common age group was 51-60 years followed by 3140 years. Out of 54 cases 37 cases were infiltrating ductal carcinoma of breast [Fig- 4a], 9 cases were squamous cell carcinoma [Fig- 4b] and 8 cases were adenocarcinoma. Miscellaneous lesions comprised of Kikuchi disease [Fig2c], Castleman disease and Kimura disease were diagnosed as $1.20 \%, 0.90 \%, 0.30 \%$ respectively of all cases.

Table 1: Histopathology of lymph node biopsies.

\begin{tabular}{|l|c|c|}
\hline Histological diagnosis & No. of case & \% \\
\hline 1) Reactive lymphadenitis & 175 & \\
a) Chronic non-specific lymphadenitis & 122 & \\
b) Follicular hyperplasia & 33 & \\
c) Sinus histiocytosis & 12 & \\
d) Paracortical hyperplasia & 08 & \\
\hline 2) Granulomatous Lymphadenitis & 82 & \\
a) Tuberculosis & 67 & \\
b) Cat scratch disease & 02 & \\
c) Others & 13 & \\
\hline
\end{tabular}




\begin{tabular}{|c|c|c|}
\hline Histological diagnosis & No. of case & $\%$ \\
\hline 3) Neoplastic disease & 66 & $19.93 \%$ \\
\hline a) Lymphoma & 12 & \\
\hline -Hodgkin's & 04 & \\
\hline -Non-Hodgkin's & 08 & \\
\hline b) Metastatic disease & 54 & \\
\hline 4) miscellaneous group & 8 & $2.41 \%$ \\
\hline a) Kikuchi disease & 04 & \\
\hline b) Castleman disease & 03 & \\
\hline c) Kimura disease & 01 & \\
\hline Total & 331 & $100 \%$ \\
\hline
\end{tabular}

Table 2: Age wise distribution of different types of lymph node biopsies.

\begin{tabular}{|c|c|c|c|c|c|c|c|}
\hline Age & $\begin{array}{c}\text { Reactive } \\
\text { lymphadenitis }\end{array}$ & $\begin{array}{l}\text { Granulomatous } \\
\text { Lymphadenitis }\end{array}$ & Hodgkin's & $\begin{array}{c}\text { Non } \\
\text { Hodgkin's }\end{array}$ & $\begin{array}{l}\text { metastatic } \\
\text { disease }\end{array}$ & $\begin{array}{l}\text { Miscella } \\
\text { neous }\end{array}$ & Total \\
\hline$<10$ & 5 & 3 & - & - & - & 2 & 10 \\
\hline $11-20$ & 39 & 16 & 1 & - & - & 3 & 59 \\
\hline $21-30$ & 51 & 17 & - & - & 2 & 3 & 73 \\
\hline $31-40$ & 25 & 19 & - & 2 & 15 & - & 61 \\
\hline $41-50$ & 22 & 16 & 2 & 3 & 12 & - & 55 \\
\hline $51-60$ & 24 & 08 & 1 & 2 & 16 & - & 51 \\
\hline $61-70$ & 06 & 03 & - & 1 & 5 & - & 15 \\
\hline$>71$ & 03 & - & - & - & 4 & - & 07 \\
\hline Total & 175 & 82 & 4 & 8 & 54 & 8 & 331 \\
\hline
\end{tabular}

\section{Site of lymph node biopsies}

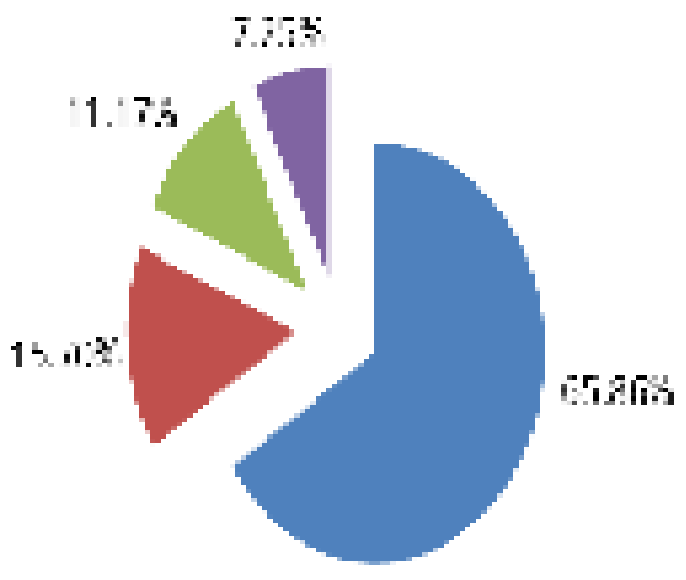

E-atial

isikn'?

Impinit

cusuning

Fig.1:-Site of lymph node biopsies. 


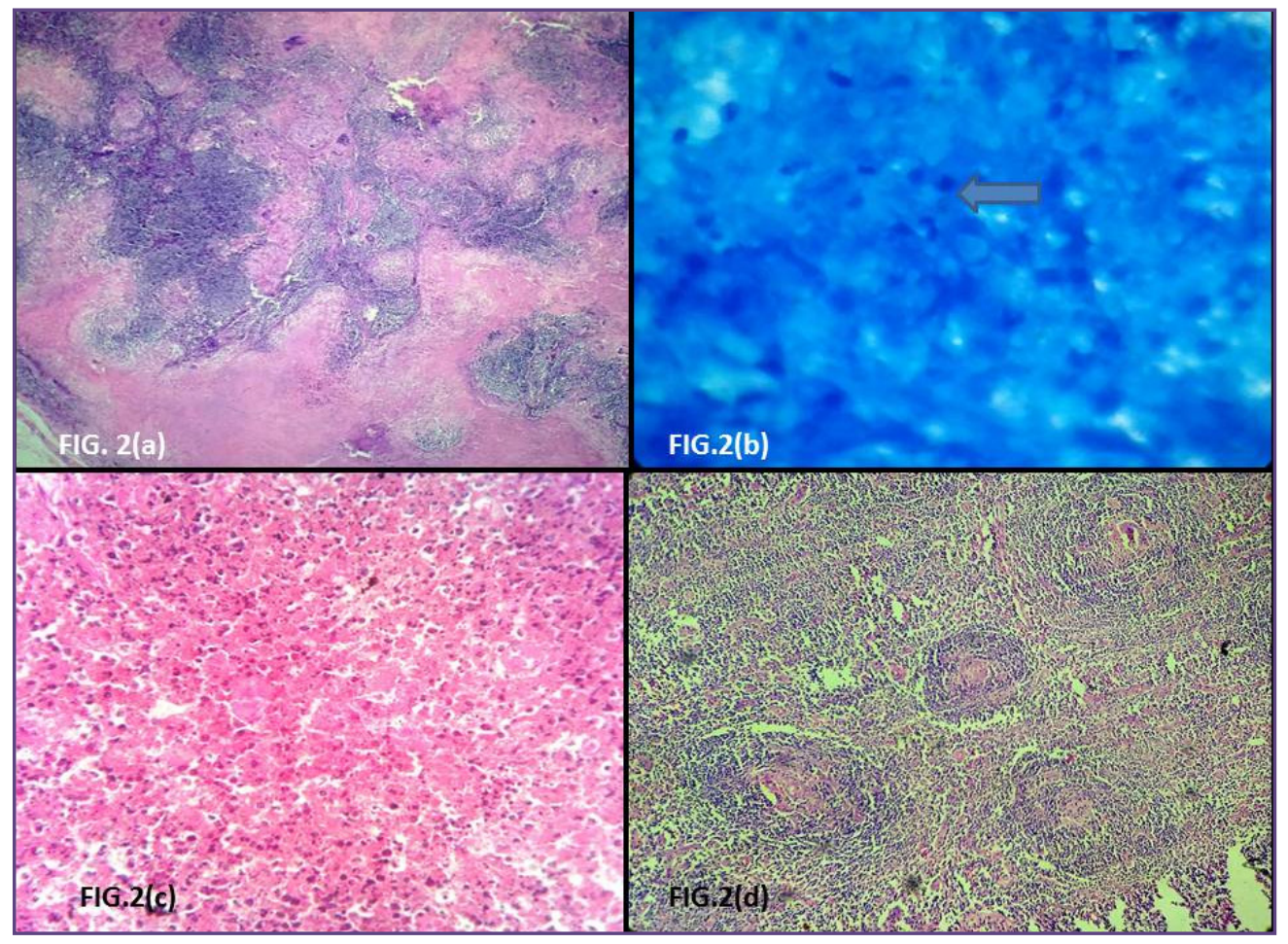

Fig. 2: Lymph node lesions (a) HPE -Tubercular Lymphadenitis showing necrotic background with epithelioid cell granuloma (H\&E; x100) (b) special stain (ZN) AFB positive bacilli (c) HPE- Kikuchi's lymphadenitis (H\&E; x400) (d) HPE- Follicular hyperplasia (H\&E; x400).

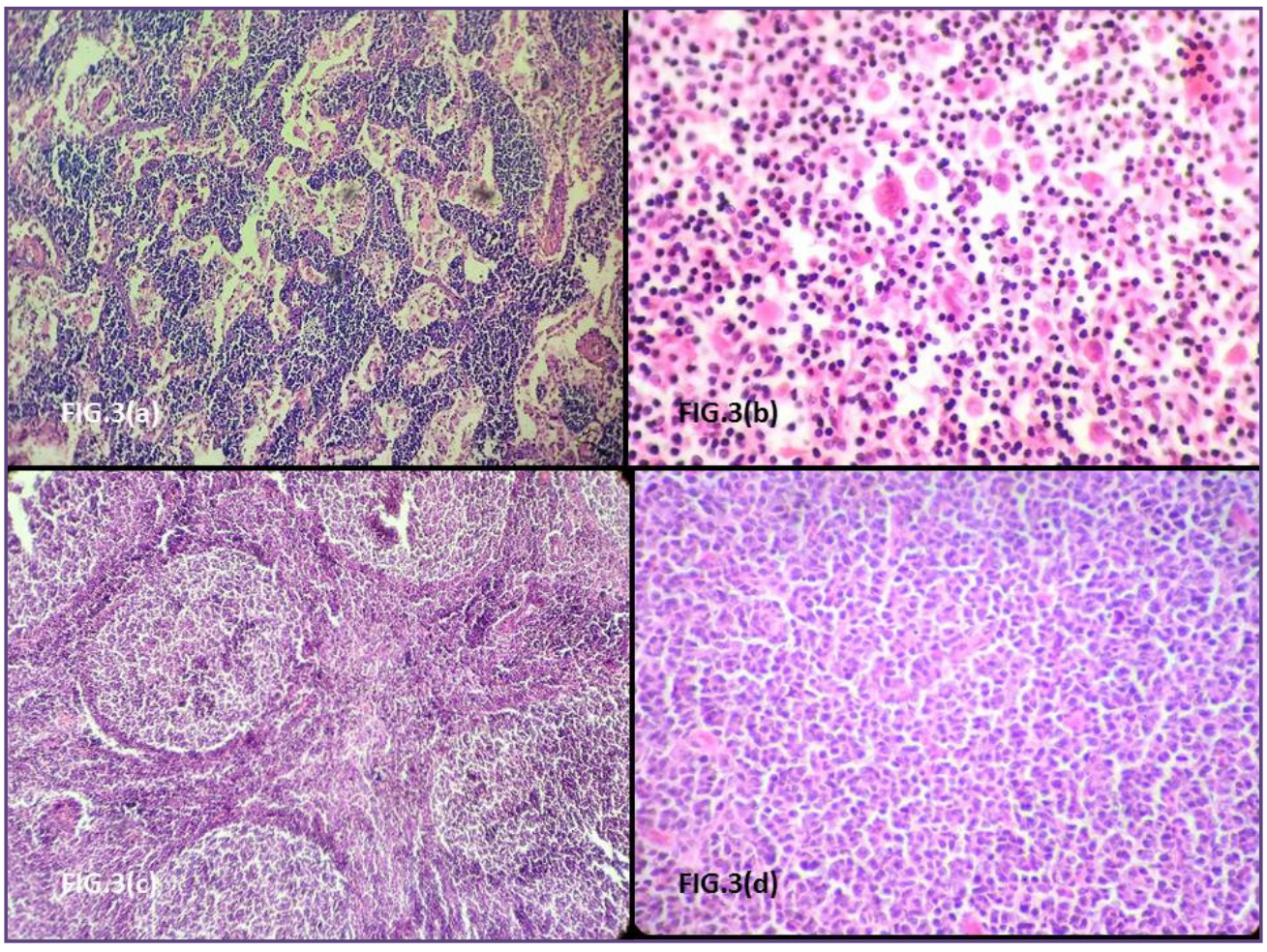

Fig. 3: Lymph node lesions (a) HPE - sinus histiocytosis (H\&E; x400) b) HPE - Hodgkin's lymphoma (H\&E; x400) (c) follicular lymphoma (H\&E; x400) and (d)HPE- diffuse small lymphocytic lymphoma (H\&E; X400). 


\section{Discussion}

Lymph node is one of the major anatomic components of the immune system. Because normal immune response leads to proliferation and expansion of one or more of the cellular components of lymph nodes, it leads to significant lymph node enlargement. ${ }^{[4]}$ Lymphadenopathy is a common clinical problem and lymph node biopsies are usually carried out to determine the cause of nodal enlargement, which may be non-neoplastic or neoplastic. Fine needle aspiration is commonly used for diagnosis of lymphadenopathy though excision biopsy is gold standard especially in suspected cases of lymphoproliferative disorders or in those cases where diagnosis cannot be reliably reached on clinical grounds or fine needle aspiration cytology.

The present study was carried out over a period of 3 years to evaluate the different histopathological patterns of lymph node biopsies and its clinicopathological correlations. In our study majority of patients were female with male to female ratio of 1:1.4 and study included patients of all age group. Similar results of female predominance were also reported by Kamat GC ${ }^{[5]}$, Tiwari $\mathrm{M}^{[6]}$, Kim LH ${ }^{[7]}$ and Rahman Md A ${ }^{[8]}$. In our study the most common clinical features were pain and tenderness which were confirmed as of infectious origin on histopathological examination.

Cervical lymph nodes are involved most often in all types of lymphadenopathy in adults as well as children. This is because these lymph nodes draining the most commonly affected and inflamed region of the body as well as due to easy accessibility of the cervical lymph node for biopsy. In our study predominant site of lymph node biopsy was cervical followed by axillary lymph nodes. This observation was consistent with most of the recent studies like Olu-

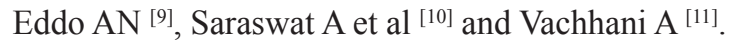

In present study non-neoplastic lesions $(80.0 \%)$ were much more common than neoplastic lesions (19.93\%) and these results were consistent with Kamat GC et al (88.92\%) ${ }^{[5]}$, Rahman Md A (70.2\%) ${ }^{[8]}$ Saraswat A et al $(90.9 \%)$ ${ }^{[10]}$, Vacchani A et al $(75 \%)^{[11]}$, and Rao MN et al $(56 \%)$ ${ }^{[12]}$. Reactive lymphadenitis $(52.87 \%)$ comprised of the predominant pattern in non-neoplastic lesions. This may be due to reactive hyperplasia in lymph nodes draining the site of malignant lesion, which do not shows metastatic deposit due to early diagnosis and surgical excision. This was consistent with study of Moore SW et al found $47.8 \%$ Of reactive lesions ${ }^{[3]}$.

Other non-neoplastic lesions comprised of (36.85\%) were chronic non-specific lymphadenitis, (9.96\%) follicular hyperplasia, (3.64\%) sinus histiocytosis and (2.41\%) of paracortical hyperplasia. Higher percentage of chronic non-specific lymphadenitis might be due to persistent bacterial, Viral and other infections in the tissue drained by the affected lymph nodes. In a study by Mohan A et al ${ }^{[13]}$ non-specific lymphadenitis was $35.6 \%$, which is consistent with the present study. Saraswat A et al ${ }^{[10]}$ reported high percentage of chronic non-specific lymphadenitis.

In a study by Reddy MP et al ${ }^{[14]}$ included 63 cases of reactive lymph nodes, 54 cases $(85.71 \%)$ were non-specific reactive hyperplasia and 9 cases $(14.29 \%)$ were follicular hyperplasia. In a study by Kamat GC et al ${ }^{[5]}$ included 75 cases of reactive lymphadenitis, 52(69.3\%) were follicular hyperplasia, $16(21.40 \%)$ were sinus histiocytosis and 7 $(9.3 \%)$ were paracortical hyperplasia.

In our study, tuberculosis was the second most common pattern constituting $20.24 \%$ of cases but several authors have reported tuberculosis as the predominant cause of lymph node enlargement. ${ }^{[5,6,8,10]}$

Among neoplastic lesions (19.93\%), there were 12 cases (3.61\%) of lymphoma. Out of which 4 cases $(1.20 \%)$ were Hodgkin's lymphoma and 8 cases $(2.41 \%)$ were NonHodgkin's lymphoma. Remaining $16.31 \%$ accounted as metastatic lesions. In a study conducted by Sibanda EN et al ${ }^{[2],}$ Kamat $\mathrm{GC}$ et a ${ }^{[5]}$ and Tiwari $\mathrm{M}$ et al ${ }^{[6]}$ reported $7 \%$, $3.6 \%$ and $2 \%$ of cases were lymphoma and which were comparable with our study. Malignancies have been the predominant cause of lymphadenitis in developed countries than developing countries like India because of racial and genetic factors. But in contrast in a study by Roy A et al [15], Sinclaire S et al ${ }^{[16]}$ and Mohan A et al ${ }^{[13]}$ constituted $44.5 \%, 63.29 \%$ and $25.9 \%$ cases of lymphoma which were very higher incidence than present study because of these studies included large number of cases and conducted in research centre or onco institute.

Metastatic malignancy were found in 54 cases $(16.31 \%)$ of which 34 cases were metastatic infiltrating breast carcinoma, 12 cases were metastatic squamous cell carcinoma and 8 cases were metastatic adenocarcinoma. Majority of cases were seen in 51-60 years followed by 31-40years of age group. In comparision to various studies in the literature our findings were similar to findings of Vachhani A et al (23\%) and Tiwari M et al (11\%) ${ }^{[11,6]}$. Metastasis of breast carcinoma in lymph node is due to adopting western lifestyle and cultural factors. Similarly squamous cell metastasis is due to consumption of tobacco in various forms in our area leading to malignancy in aerodigestive tract.

\section{Conclusion}

The study highlights the importance of lymph node biopsy for early diagnosis and establishing the cause of 
lymphadenopathy. So more attention should be given to lymph node swelling for the appropriate clinical management of patients. Reactive lymphadenitis was the most common cause of lymphadenopathy followed by granulomatous lymphadenitis in our study.

\section{References}

1. Robbins S, Cotran R. Diseases of the immune system. In: Kumar V, Abbas AK, Sausto N, Aster JC (eds). Robbins and Cotran pathologic basis of disease.8th edn. Philadelphia: Elsevier Saunders, 2010: 235-249.

2. Sibanda EN, Stanczuk G. Lymph node pathology in Zimbabwe: A review of 2194 specimens. Q J Med 1993;86:811-7.

3. Moore SW, Schneider JW, Schaaf HS. Diagnostic aspects of cervical lymphadenopathy in children in the developing world: A study of 1877 surgical specimens. Pediatr Surg Int 2003;19:240-4.

4. Longo, Fuci et al, Harrison's Internal Medicine 17th edition. Ch-60, p-370-372.

5. Kamat GC. A ten-year histopathological study of generalized lymphadenopathy in India. S Afr Fam Pract 2011; 53(3): 267-270.

6. Tiwari M, Aryal G, Shrestha R. Histopathologic diagnosis of lymph node biopsies. Nepal Med Coll J2007;9(4) : 259-61.

7. Kim LH, Peh SC, Chan KS. Pattern of lymph node pathology in a private pathology laboratory. Malays J Pathol 1999 ;21(2) :87-93.
8. Md Atiqur R, Md Mamun AB. Histopathological evaluation of lymph node biopsies: A hospital based study. J Enam Med Col $2012 ; 2(1): 8-14$.

9. Olu-Eddo AN, Ohanaka CE. Peripheral lymphadenopathy in Nigerian adults.J Pak Med Assoc 2006 ;56 : 405-8.

10. Saraswat A, Rajender A, Purohit K. Lymph node biopsy: Spectrum and clinical significance as diagnostic tool at tertiary care centre.J of Evolution Med and Dent Sci. 2015; 4(6): 1008-14.

11. Vachhani A, Bhuva K, Jasani J, et al. Histopathological study of lymph node biopsy. International Journal of Biomedical and Advance Research 2013; 4 (11) : 790-5.

12. Rao MN, Raju YS, Prasad AK, et al. Evaluation of lymphadenopathy at a referral centre. JAPI ; 50 : 1488-1489.

13. Mohan A, Reddy MK, Phaneendra BV, Chandra A. Aetiology of peripheral lymphadenopathy in adults: analysis of 1724 cases seen at a tertiary care teaching hospital in Southern India. Natl Med J India 2007; 20(2): 78-80.

14. Reddy MP, Moorchung N, Choudhary A. Clinicopathological profile of pediatric lymphadenopathy. Ind J Paediatr 2002; 69 : 1047-1051.

15. Roy A, Kar R, Basu D, Badhe BA. Spectrum of histopathologic diagnosis of lymph node biopsies: a descriptive study from a tertiary care center in South India over $5 \frac{1}{2}$ years. Indian J Pathol Microbiol 2013; 56(2) : 103-8.

16. Sinclair S, Beckman E, Ellman L. Biopsy of enlarged superficial lymph nodes. JAMA 1974; 228(5): 602-603.

*Corresponding author:

Dr. Rajshri P. Damle, Department Of Pathology, A.C.P.M. Medical College, Dhule, Maharashtra. India. 424005

Phone: +91 9767637624

Email: rajshriborase@gmail.com

Date of Submission : 04.10.2016

Date of Acceptance : 21.01.2017

Financial or other Competing Interests: None.
Date of Publication : 02.04.2017 